\title{
On the continuum limit of Landau gauge gluon and ghost propagators in $S U(2)$ lattice gauge gluodynamics
}

\author{
Igor Bogolubsky* \\ Joint Institute for Nuclear Research, LIT, 141980 Dubna, Russia \\ E-mail: bogolubsejinr.ru
}

\section{Ernst-Michael Ilgenfritz}

Joint Institute for Nuclear Research, VBLHEP, 141980 Dubna, Russia

E-mail: michael.ilgenfritzelhep.jinr.ru

\section{Michael Müller-Preussker}

Humboldt-Universität zu Berlin, Institut für Physik, 12489 Berlin, Germany

E-mail: mmp@physik.hu-berlin.de

\section{Andre Sternbeck}

Universität Regensburg, Institut für Theoretische Physik, 93040 Regensburg, Germany

E-mail: andre.sternbeck@physik.uni-regensburg.de

\begin{abstract}
We continue the systematic computation of Landau gauge gluon and ghost propagators of $S U(2)$ gluodynamics using a sequence of increasing lattice sizes $L^{4}$ up to $L=112$ with corresponding $\beta$-values chosen to keep the linear physical size $a(\beta) L \simeq 9.6 \mathrm{fm}$ fixed. To extremize the Landau gauge functional we employ simulated annealing combined with subsequent overrelaxation. Renormalizing the propagators at momentum $\mu=2.2 \mathrm{GeV}$ we observe quite strong lattice artifacts for the gluon propagator as well as for the ghost dressing function within the momentum region $q<1.0 \mathrm{GeV}$. The dependence on the lattice spacing for the gluon propagator at lowest accessible physical momentum values does not yet allow a simple extrapolation to the continuum limit. On the contrary, the running coupling derived from the bare dressing functions seems less affected by lattice artifacts.
\end{abstract}

Xth Quark Confinement and the Hadron Spectrum,

October 8-12, 2012

TUM Campus Garching, Munich, Germany

\footnotetext{
*Speaker.
} 


\begin{tabular}{cccccccc}
\hline$\beta$ & 2.3 & 2.3 & 2.3 & 2.3 & 2.4 & 2.45 & 2.5 \\
\hline$L$ & 40 & 56 & 80 & 112 & 80 & 96 & 112 \\
\hline$N_{M C}$ & 45 & 187 & 78 & 173 & 314 & 333 & 477 \\
$N_{\text {ghost }}$ & - & 24 & - & - & 26 & 1 & - \\
$\tilde{Z}(\mu=2.2 \mathrm{GeV})$ & - & $0.414(3)$ & - & - & $0.445(4)$ & $0.452(2)$ & $0.460(5)$ \\
\hline
\end{tabular}

Table 1: Parameters of the main simulations considered here. See also the text.
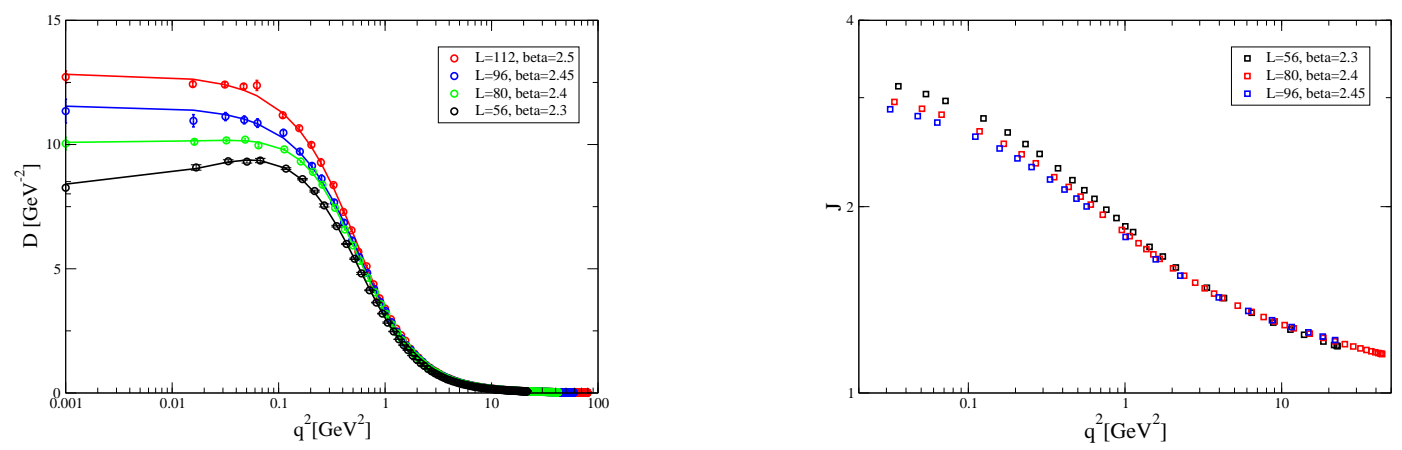

Figure 1: Left: the unrenormalized gluon propagator $D\left(q^{2}\right)$ and its fits according to Eq. (1); the data points drawn at $q^{2}=0.001$ represent the zero-momentum gluon propagator $D(0)$ values. Right: the ghost dressing function $J\left(q^{2}\right)$. Both are shown for approximately equal physical volume ( $\left.(a L)^{4} \simeq(9.6 \mathrm{fm})^{4}\right)$ but different $\beta$-values, i.e. discretization scales $a$.

The aim of the reported study is to continue the systematic investigation of $S U(2)$ gluon and ghost Landau gauge propagators on large lattices [1] in order to receive information from first principles on the behavior of these propagators and of the running coupling in the continuum limit for all momenta $q$ including the infrared (IR) region. As in previous investigations [2], for solving the Gribov problem we assume the Landau gauge functional to be driven as close as possible to its global extremum. Employing the standard Wilson plaquette action we have studied gluon $(D)$ and ghost $(G)$ propagators for lattice sizes $L^{4}$ with run parameters collected in Table 1 . The gluon propagator was computed using $N_{M C}(\beta, L)$ independent Monte Carlo (MC) configurations generated with the given set of parameters, while the ghost propagator was calculated only on a subset of $N_{\text {ghost }}(\beta, L)$ MC configurations. We used the same procedures for Landau gauge fixing and computation of propagators as described in [1], namely, we employed very long simulated annealing (SA) runs followed by overrelaxation (OR) to obtain gauge copies with a gauge fixing functional close to its global extremum for each MC configuration. In Fig. 1 we show the results for the bare gluon propagator (left) and the bare ghost dressing function (right) for fixed physical volume $(a L)^{4}$ but varying lattice scale $a$. For the gluon propagator we have drawn also curves 


\begin{tabular}{cccccccc}
\hline$\beta$ & $C$ & $A$ & $B$ & $E$ & $F$ & $G$ & $\chi^{2} /$ dof \\
\hline 2.3 & $1.164(3)$ & $1.52(1)$ & $0.11(3)$ & $0.68(2)$ & $0.502(4)$ & $0.126(15)$ & 1.03 \\
\hline 2.4 & $1.195(2)$ & $1.68(2)$ & $1.1(3)$ & $1.13(10)$ & $0.60(3)$ & $0.36(5)$ & 2.73 \\
\hline 2.45 & $1.148(11)$ & $3.9(5)$ & $99(34)$ & $22(7)$ & $12(4)$ & $3.1(5)$ & 0.28 \\
\hline 2.5 & $1.127(8)$ & $5.47(34)$ & $210(33)$ & $45(6)$ & $25(4)$ & $4.3(3)$ & 1.20 \\
\hline
\end{tabular}

Table 2: Results of the 6-parameter fits of the unrenormalized gluon propagator for various $\beta$ corresponding to lattices sizes $56^{4}, 80^{4}, 96^{4}$ and $112^{4}$.

obtained from fits with the 6-parameter formula proposed in [3]

$$
D(q)=C \frac{q^{4}+A^{2} q^{2}+B}{q^{6}+E q^{4}+F q^{2}+G^{2}} .
$$

We found the resulting fit curves nicely to capture the IR turnover of the gluon propagator. The $\chi^{2} / d o f$ values are close to unity in most cases (see Table 2). To obtain the renormalized gluon propagator $D_{r e n}(q, \mu)=\tilde{Z}(a, \mu) D(q, a)$ we apply the normalization condition $D_{\text {ren }}(\mu, \mu)=1 / \mu^{2}$. Since the fit formula Eq. (1) nicely works throughout the whole momentum region we can use it to carry out the renormalization at any $\mu$. For $\mu=2.2 \mathrm{GeV}$ the renormalization factors $\tilde{Z}$ are collected in Table 1 . Their values do not vary strongly which means that the bare dressing functions for the different $\beta$-values approximately overlap at the given $\mu$-value. From Fig. 1 we can then conclude also for the renormalized gluon propagator $D_{r e n}\left(q^{2}\right)$ and the renormalized ghost dressing function $J_{\text {ren }}\left(q^{2}\right)$ found for various lattice spacings $a(\beta)$ to be compatible with the so-called decoupling solution of Dyson-Schwinger or functional renormalization group equations (see [4]). The numerical values, however, of $D_{r e n}\left(q^{2}\right)$ and $J_{r e n}\left(q^{2}\right)$ in the limit $q \rightarrow 0$ appear to be $\beta$ - or $a$-dependent. From such plots one can see that the convergence of the renormalized lattice propagators / dressing functions in the deep IR momentum range to the respective continuum counterpart, that should be observed for decreasing $a(\beta)$, is rather slow. For their direct numerical study near the continuum limit one has to use rather large $\beta$-values which consequently requires simulations on unrealistically huge lattices, which are not accessible today even on most powerful parallel supercomputers. Instead, we can try to make contact with the continuum limit by extrapolating $D_{\text {ren }}\left(q^{2}, a\right)$ to the zero- $a$ limit as done e.g. in [5] for $S U(3)$ and non-zero temperature. In Fig. 2 (left) we plot the $a$-dependence of lattice $D_{\text {ren }}\left(q^{2}\right)$ for several selected values of $q^{2}$. We see the lower the momentum is the less well-defind the convergence for $a \rightarrow 0$ becomes. For getting reliable numerical values of $S U(2)$ gluon and ghost propagators in the continuum limit more work is needed. Although the ghost dressing function $J\left(q^{2}\right)$ has been computed only for a subset of MC configurations (see Table 2), it provided useful quantitative information, see Fig. 1 (right). Even a single MC configuration as for $L=96$ already seems to yield a first estimate (a fast decrease of the statistical fluctuations of $J\left(q^{2}\right)$ with increasing $L$ was first observed for the $S U(3)$ case in [6]). Our analysis shows in the deep IR region that, while $D_{\text {ren }}\left(q^{2}\right)$ increases with $\beta, J_{\text {ren }}\left(q^{2}\right)$ decreases. More details will be published elsewhere. 

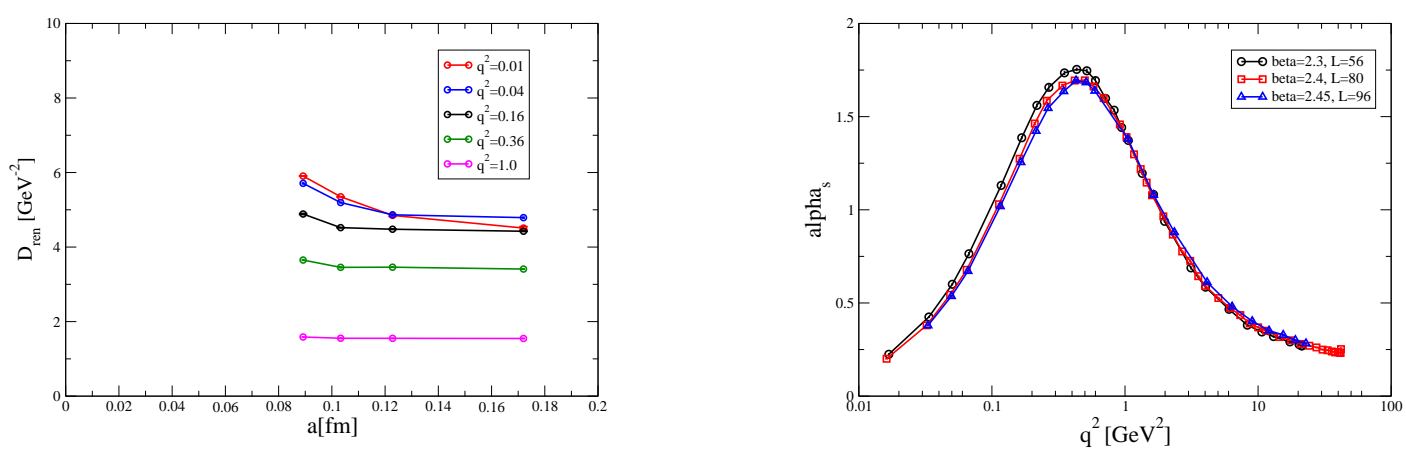

Figure 2: Left: Renormalized gluon propagator $D_{r e n}\left(q^{2}\right)$ at $\mu=2.2 \mathrm{GeV}$ versus lattice spacing $a$ for various physical momenta $q^{2}$ (values indicated in units $\mathrm{GeV}^{2}$ ). Right: Running coupling for $\beta=2.3, \beta=2.4$ and $\beta=2.45$
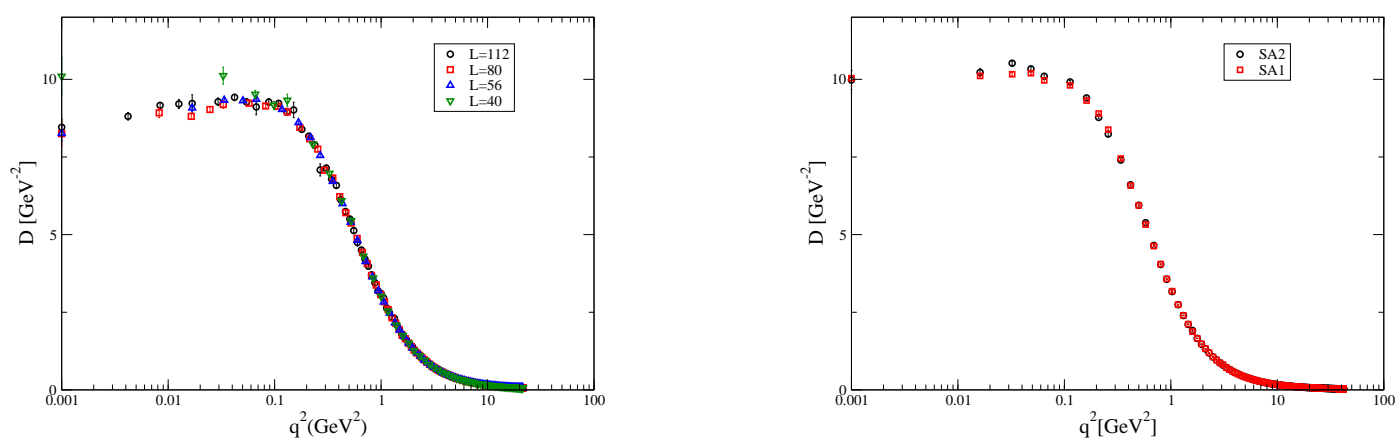

Figure 3: Check of systematic errors. Left: The unrenormalized $D\left(q^{2}\right)$ computed for $\beta=2.3$ and various $L$. Right: $D\left(q^{2}\right)$ for 2 different SA schedules at $\beta=2.4$ and $L=80$.

We have checked whether the differences of propagator values in the deep IR could be compensated by other systematic effects. From Fig. 3 (left) one can see that finite-volume effects are small if the linear physical size is $a(\beta) L \simeq 9.6 \mathrm{fm}$ or even larger. What concerns Gribov copy artifacts at $\beta=2.4$ and $L=80$ we have compared the results of two sets of $\mathrm{SA}+\mathrm{OR}$ gauge fixing simulations: (i) one gauge copy fixing with 9600 SA sweeps ("SA1 schedule") $\left(N_{M C}=314\right)$ and (ii) "best of two copies" gauge fixing with 12000 SA sweeps each ("SA2 schedule") $\left(N_{M C}=187\right)$. For details see, e.g., Ref. [7]. SA1 and SA2 results obtained for unrenormalized gluon propagators are plotted in Fig. 3 (right). We have found that the differences between these cases are much smaller than the magnitude of Gribov copy effects measured in [1] as difference between results of one-copy SA+OR and one-copy OR gauge-fixing procedures. Our analysis shows that further "improvement" of SA schedules could not change $D_{r e n}\left(q^{2}\right)$ essentially and hence noticeable differences of $D_{\text {ren }}\left(q^{2}\right)$ values in the deep IR region found for different $\beta$-values certainly cannot be accounted for by the Gribov copy effect. 
With the bare gluon $\left(Z\left(q^{2}\right)\right)$ and ghost $\left(J\left(q^{2}\right)\right)$ dressing functions at hand one can easily compute the running coupling in the minimal MOM scheme [8],

$$
\alpha_{s}\left(q^{2}\right)=\frac{g_{0}^{2}}{4 \pi} J^{2}\left(q^{2}\right) Z\left(q^{2}\right)
$$

The dependence of the resulting curves on $\beta$ or $a$ turns out to be rather weak even in the deep IR momentum region (see Fig. 2 (right)), i.e. the lattice artifacts of the gluon and ghost dressing functions cancel each other to some extent.

We conclude that naive multiplicative renormalizability for the $S U(2)$ Landau gauge gluon and ghost propagators gets violated in the deep IR region. Due to the slow convergence of gluon and ghost renormalized propagators their continuum counterparts may strongly differ in the deep IR momentum region from what we have obtained here in lattice simulations with admissible values of $\beta=4 / g_{0}^{2}$. At the same time, the physically important renorm-invariant minimal MOM-scheme running coupling $\alpha_{s}\left(q^{2}\right)$ seems to reach a continuum behavior much earlier.

IB thanks Prof. A. A. Slavnov for a useful discussion of the results. Simulations have been done on the MVS100K supercomputer of the Joint Supercomputer Centre (JSCC, Moscow).

\section{References}

[1] I. L. Bogolubsky, E.-M. Ilgenfritz, M. Müller-Preussker, and A. Sternbeck, PoS (LAT2009) 237 (2009), arXiv:0912.2249 [hep-lat] .

[2] T. D. Bakeev, E.-M. Ilgenfritz, V. K. Mitrjushkin, and M. Müller-Preussker, Phys. Rev. D69, 074507 (2004), hep-lat/0311041 ; I. L. Bogolubsky, G. Burgio, V. K. Mitrjushkin, and M. Müller-Preussker, Phys. Rev. D74, 034503 (2006), hep-lat/0511056 ; I. L. Bogolubsky, V. G. Bornyakov, G. Burgio, E.-M. Ilgenfritz, V. K. Mitrjushkin, and M. Müller-Preussker, Phys. Rev. D77, 014504 (2008), arXiv:0707.3611 [hep-lat] ; V. Bornyakov, V. Mitrjushkin, and M. Müller-Preussker, Phys. Rev. D81, 054503 (2010), arXiv:0912.4475 [hep-lat] .

[3] A. Cucchieri, D. Dudal, T. Mendes, and N. Vandersickel, Phys.Rev. D85, 094513 (2012), arXiv:1111.2327 [hep-lat] .

[4] C. S. Fischer, A. Maas, and J. M. Pawlowski, Annals Phys. 324, 2408 (2009), arXiv:0810.1987 [hep-ph] .

[5] R. Aouane, V. G. Bornyakov, E.-M. Ilgenfritz, V. K. Mitrjushkin, M. Müller-Preussker, and A. Sternbeck, Phys.Rev. D85, 034501 (2012), arXiv:1108.1735 [hep-lat] .

[6] I. L. Bogolubsky, E.-M. Ilgenfritz, M. Müller-Preussker, and A. Sternbeck, PoS LAT2007, 290 (2007), arXiv:0710.1968 [hep-lat] .

[7] I. L. Bogolubsky, E.-M. Ilgenfritz, M. Müller-Preussker, and A. Sternbeck, Phys. Lett. B676, 69 (2009), arXiv:0901.0736 [hep-lat] .

[8] L. von Smekal, K. Maltman, and A. Sternbeck, Phys. Lett. B681, 336 (2009), arXiv:0903.1696 [hep-ph] . 